\title{
Unexpected Perinatal Loss versus Sids - a Common Neuropathologic Entity
}

\author{
Luigi Matturri*, $^{*}$, Maria Mauri ${ }^{1}$, Maria Elena Ferrero ${ }^{2}$ and Anna Maria Lavezzi ${ }^{1}$ \\ 1 "Lino Rossi" Research Center for the study and prevention of unexpected perinatal death and SIDS, University of Mi- \\ lan, Italy and ${ }^{2}$ Institute of General Pathology, University of Milan, Italy
}

\begin{abstract}
Objective: To evaluate the involvement of alterations of the central autonomic nervous system, particularly of the brainstem and cerebellum, in a wide set of victims of sudden and unexplained perinatal and infant death.

Material and Methods: The study population consisted of 63 stillbirths, 28 neonatal deaths and 140 suspected SIDS. The victims were subjected to in-depth anatomopathological examination following appropriate guidelines. The protocol included, in particular, the histological evaluation on serial sections of the cardiorespiratory autonomic nervous system.

Results: A diagnosis of "unexplained death" was established for 217 of the 231 victims (59 stillbirths, 28 newborns and 130 SIDS). In a very high percentage of these deaths (84\%) we observed one or more anomalies of the nuclei and/or structures of the brainstem and cerebellum related to vital functions.
\end{abstract}

Conclusion: Unexpected perinatal loss should not be regarded as a separate entity from SIDS, given the common neuropathological substrates.

Keywords: Stillbirth, SIDS, Central Autonomic Nervous System, Brainstem, Neuropathology, Developmental Alterations.

\section{INTRODUCTION}

Sudden perinatal death (that includes "ante-partum death", from 22 completed gestational weeks, "intra-partum death" and "early neonatal death" occurring during the first seven completed days of life) and sudden infant death syndrome (SIDS) represent facets of a multifactorial problem that has not yet found a univocal approach on the clinical plane [1-6]. Despite the efforts of many investigators, the true causes of unexpected stillbirth and SIDS remain unknown. This is due to a lack of appropriately detailed postmortem studies.

A fundamental component of these deaths is neuropathology. In fact, pathogenesis of unexplained perinatal death and SIDS seems to privilege, in most cases, some nervous dysfunctions. Nevertheless, only a few studies in this field have adequately examined the neurological substrates, although even subtle abnormalities of structures of the autonomic nervous system can determine dysfunctions in the control of vital functions, leading to unexpected perinatal loss as well as infant death [7-10].

The present study will focus upon the multifaceted involvement of the central autonomic nervous system, particularly of the brainstem and cerebellum, in the disruption of neuronal pathways observed in a large population of sudden and unexplained perinatal and infant death victims.

*Address correspondence to this author at the "Lino Rossi" Research Center for the study and prevention of unexpected perinatal death and SIDS, University of Milan, Via della Commenda, 19, 20122 Milan, Italy; Tel: +39-0250320800; Fax: +39-02-50320823; E-mail: luigi.matturri@unimi.it

\section{MATERIAL AND METHODS}

Our study was focused on 231 victims of sudden and unexplained perinatal and infant death, aged from 22 gestational weeks to 10 postnatal months. This was a selected set of cases, sent to our Center over a 6-year period (2000-2006) on the basis of a specific decree passed by the Lombardy Region [11]. This decree specifies that all cases of unexplained perinatal death and/or of suspected SIDS must be sent to the L. Rossi Center of Milan University, being the Regional Reference Center, for in-depth anatomopathological examination, in accordance with specific guidelines [12-14]. This protocol includes, in particular, the histological evaluation on serial sections of the cardiorespiratory autonomic nervous system, besides the examination of the placental disk, umbilical cord and membranes in fetuses.

For every case, a complete clinical history, particularly referred to the mother in perinatal deaths, and including the death scene examination in infant deaths, was collected. Cases were number-coded and identifying data (name, address, etc.) were removed prior to analysis.

The study population consisted of 63 stillbirths, 28 neonatal deaths and 140 suspected SIDS.

The 63 fetuses, 36 males and 27 females, were aged from 22 to 41 gestational weeks, with a peak incidence at 36 to 38 weeks $(62 \%)$. The weights of victims ranged from 352 to 3500 grams, being below the corresponding reference value in most cases.

The 28 newborns, 16 males and 12 females, died intrapartum or in the first week after birth. 
Table 1. Case Profiles of the Study

\begin{tabular}{|c|c|c|c|c|c|}
\hline \multirow{2}{*}{ Victims } & \multirow{2}{*}{ Age (range) } & \multicolumn{2}{|c|}{$\operatorname{Sex}(n)}$. & \multicolumn{2}{|r|}{ Death Diagnosis } \\
\hline & & $\mathbf{M}$ & $\mathbf{F}$ & Unexplained deaths (n.217) & Border line deaths (n.14) \\
\hline $\begin{array}{c}\text { Fetuses } \\
\text { (n.63) }\end{array}$ & $22-41 \mathrm{gw}$ & 36 & 27 & unexplained stillbirth (n.59) & Annexa anomalies (n.4) \\
\hline Newborns (n.28) & $1-7 \mathrm{pd}$ & 16 & 12 & unexplained neonatal death (n.28) & - \\
\hline Infants (n.140) & $1-10 \mathrm{~m}$ & 88 & 52 & SIDS (n.130) & $\begin{array}{l}\text { Neoplastic lesions of the cardiac conduction system (n.3) } \\
\text { Brainstem encephalitis + moderate myocarditis (n.4) } \\
\text { Tractus solitarii nucleus necrosis (n.1) } \\
\text { Area postrema hemangioendotelioma (n.1) } \\
\text { Pneumonia + hypoglossal nucleus hypoplasia (n.1) }\end{array}$ \\
\hline
\end{tabular}

$\mathrm{gw}=$ gestational week; $\mathrm{pd}=$ postnatal day $; \mathrm{m}=$ month SIDS = Sudden Infant Death Syndrome.

Among the infant victims, 52 females and 88 males, sudden death occurred between 1 and 10 months of life, with the greatest incidence around the third month.

After an in-depth autopsy and histological examination, a diagnosis of "unexplained death" was established for 217 of the 231 victims, namely 59 stillbirths, 28 newborns and 130 infants, all clearly SIDS victims.

The remaining 14 cases (4 fetal and 10 infant deaths) $(6 \%)$, were classified as "border-line", since it was difficult to establish whether the pathological findings were sufficiently severe to have caused the death. In the infant deaths we observed: neoplastic lesions of the cardiac conduction system, i.e. Purkinje cell tumor and cardiac fibroma in 3 cases; encephalitic features (probably viral in etiology) in nuclei of the brainstem and moderate myocarditis in 4 cases; a case with focal necrosis of the tractus solitarii nucleus, a case with capillary hemangioendothelioma in the area postrema and a case with pneumonia associated to hypoglossal nucleus hypoplasia. In four stillbirths, abnormalities of the annexa were diagnosed (umbilical cord around the neck, head band of the umbilical cord, multifocal placental infarcts). Table 1 summarizes the case profiles of the study.

None of the mothers had any significant pathology. With reference to smoking habit, 81 mothers $(35 \%)$ declared that they were active smokers (all of more than 3 cigarettes/day and all already before becoming pregnant) while 129 (56\%) were non smokers. In 21 cases no information about smoking habit was available.

The focus of this study is the in-depth histological examination of the central autonomic nervous system. Hereafter, we will briefly describe the study protocol. More detailed information is available on the previous publications [12-14].

The brainstem and cerebellum, where the main vital centers are located, were processed and embedded in paraffin. Transverse serial sections were made at intervals of $30 \mu \mathrm{m}$. For each level, twelve $5 \mu \mathrm{m}$ sections were obtained, two of which were routinely stained for histological examination using alternately hematoxylin-eosin and Klüver-Barrera stains. Two additional sections at each level were subjected to immunohistochemistry for the study of neurotransmitters (somatostatin and tyrosine hydroxylase $(\mathrm{TH})$, indicative of catecholamine synthesis) and apoptosis. The remaining sections were saved and stained as deemed necessary for further investigations.

The main nuclei were analyzed in serial sections of the brainstem, namely the parafacial nucleus, the locus coeruleus and the parabrachial/Kölliker-Fuse complex in the pons and mesencephalon; the hypoglossus, the dorsal motor vagal, the tractus solitarius, the ambiguus, the pre-Bötzinger, the inferior olivary and the arcuate nuclei in the medulla oblongata. Fig. (1) shows the sampling of the brainstem (three specimens are obtained: the first, ponto-mesencephalic, includes the upper third of the pons and the adjacent portion of mesencephalon; the second extends from the rostral limit of the medulla oblongata to the adjacent caudal pons, and the third specimen takes as reference point, the obex and extends 2-3 $\mathrm{mm}$ above it and below it). Fig. (2) shows the histological sections corresponding to the three brainstem samples of Fig. (1) indicating the main nuclei and structures to be examined.

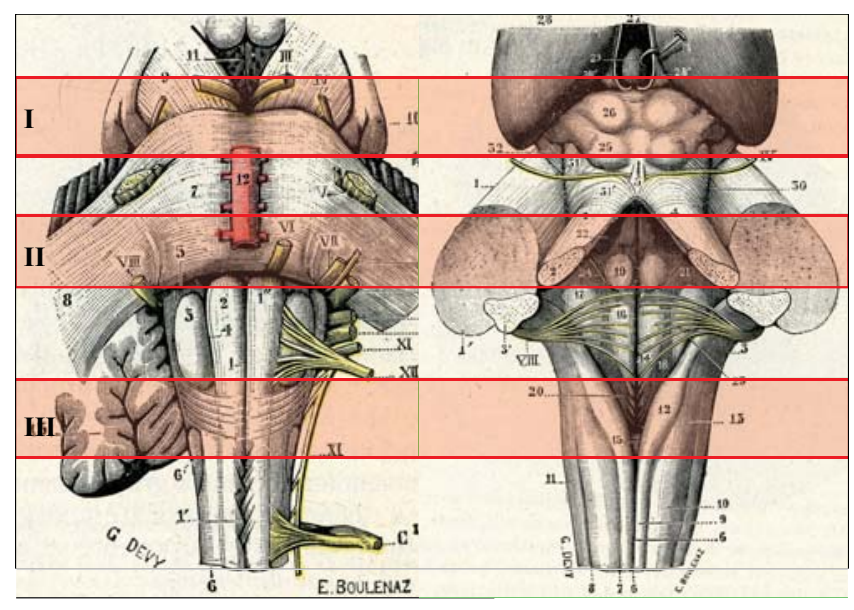

Fig. (1). Sampling of the brainstem: ventral (left) and dorsal (right) surface.

In the cerebellum, the cortex layers (external granular layer, molecular layer, Purkinje cell layer and internal granular layer) and the medullary deep nuclei (the dentate nucleus, the fastigial nucleus, the globose nucleus and the emboliform nucleus) were examined. 


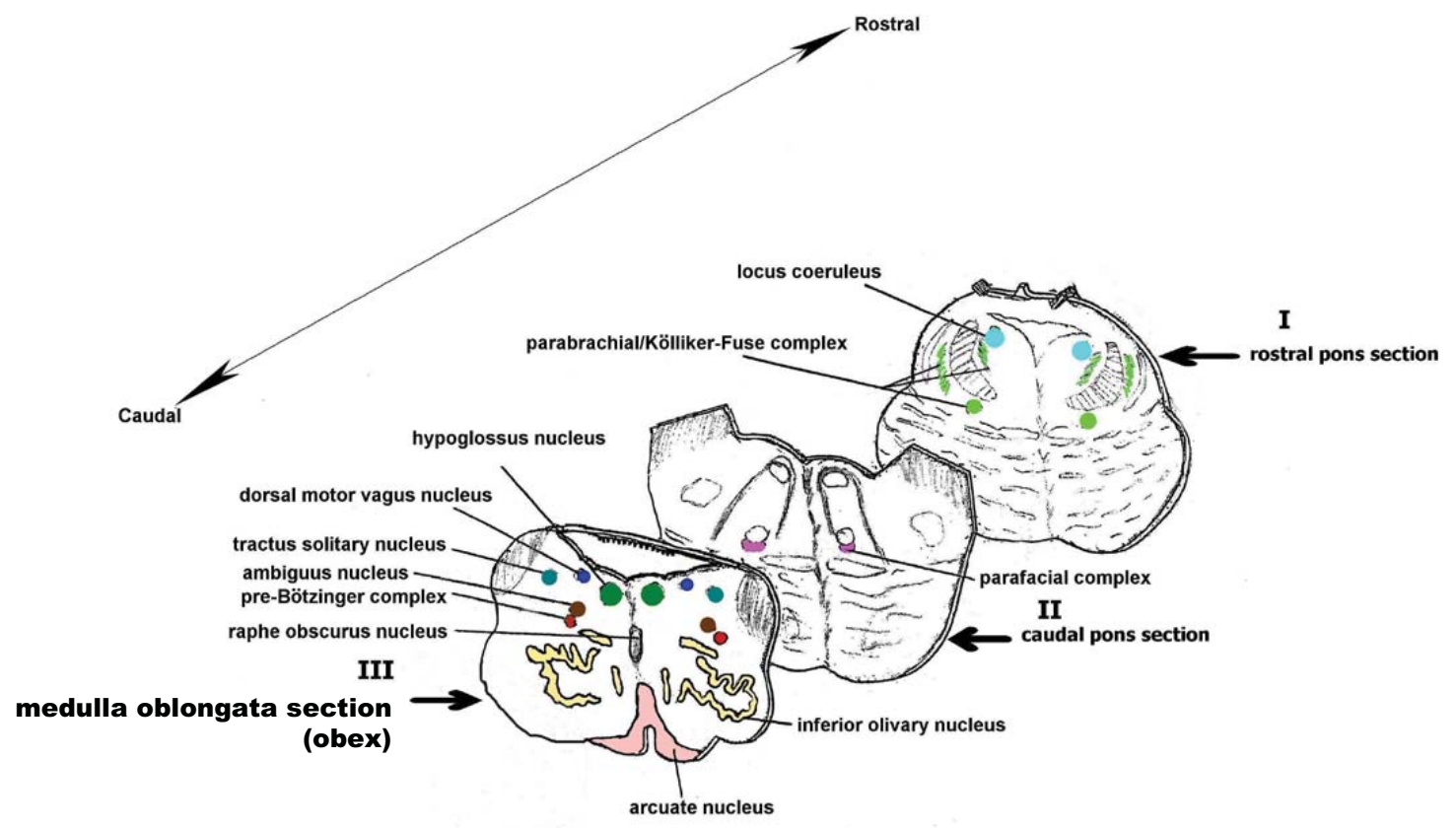

Fig. (2). Schematic representation of the main histological sections obtained from the brainstem for the anatomopathologic examination.

\section{Immunohistochemical Methods}

In order to analyze the immuno-expression of neurotransmitters, particularly of somatostatin and catecholamines, we used specific primary antibodies after applying the avidin-biotin-peroxidase technique, in conformity with conventional immunohistochemical procedures. Immunohistochemical visualization of apoptotic cells was obtained by the TUNEL method (TdT-mediated dUTP-biotin nick end labeling). A detailed description of the immunohistochemical procedures that we applied, including the immunopositivity evaluation, is available in our previous works [15-17].

\section{Statistical Analysis}

The statistical significance of direct comparison between groups of victims (unexplained fetal, neonatal and infant deaths, border-line cases) was determined using one way analysis of variance (ANOVA) and Student's paired t-test. The selected threshold level for statistical significance was $\mathrm{p}<0.05$.

\section{RESULTS}

The in-depth anatomo-pathological examination of the central autonomic nervous system performed in the 217 victims (59 stillbirths, 28 newborns and 130 SIDS) of unexplained perinatal and infant death, revealed one or more anomalies of the nuclei and/or structures of the brainstem and cerebellum related to vital functions, mainly to cardiorespiratory control, in 182 cases $(84 \%)$.

\section{Main Morphological Findings in Brainstem and Cerebel- lum}

\section{Medulla Oblongata}

Hypoplasia of the arcuate nucleus emerged in $56 \%$ of the sudden fetal deaths (33 cases) and in $50 \%$ of both unex- plained neonatal deaths (14 cases) and in SIDS victims (65 cases). Different degrees of hypodevelopment were observed, ranging from a normal structure with neuronal immaturity to total agenesis of the nucleus, observed in 8 stillbirths (Fig. 3).

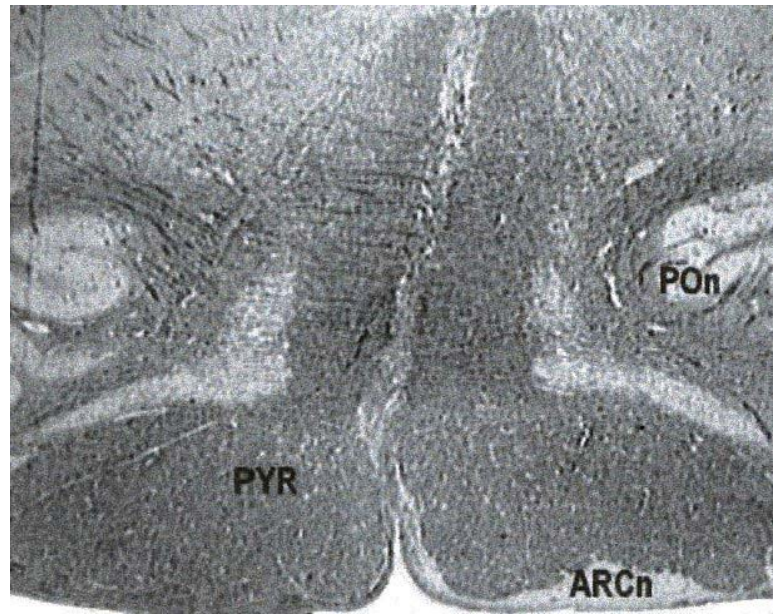

Fig. (3). Transversal section of medulla oblongata with severe hypoplasia of the arcuate nucleus (magnification: 10x)

Abbreviations: ARCn, arcuate nucleus; POn, principal inferior olivary nucleus; PYR, pyramid.

Hypoplasia of the pre-Bötzinger nucleus, with a decreased number of neuronal bodies and/or dendritic fibers, was diagnosed in $25 \%$ of stillbirths (15 cases) and in $8 \%$ of SIDS (10 cases).

\section{Pons-Mesencephalon}

In transverse sections of rostral pons and caudal mesencephalon of 14 unexplained stillbirths (24\%) and of 8 unex- 
plained neonatal deaths $(30 \%)$, prevalently intra-partum deaths, a very few immature neurons were observed in both the medial and lateral parabrachial nuclei and in the Kölliker-Fuse area; altogether, these findings led to a diagnosis of hypoplasia of the parabrachial/Kölliker-Fuse complex.

Hypoplasia of the parafacial nucleus, with a decreased neuronal density and transverse area, was diagnosed in the caudal pons of 41 stillbirths $(70 \%$ of unexplained fetal deaths) but in no case of post-natal death.

\section{Cerebellum}

Histological examination revealed morphological alterations of the cortex development. In fact, the cerebellar cortex showed an immature structure, uniformly made up of small round cells without the usual four-layered shape, in $10 \%$ of the unexplained stillbirths ( 6 cases, all after the $36^{\text {th }}$ gestational week), in 6 neonatal deaths (20\%) and in $26 \%$ of the SIDS victims (34 cases) (Fig. 4).

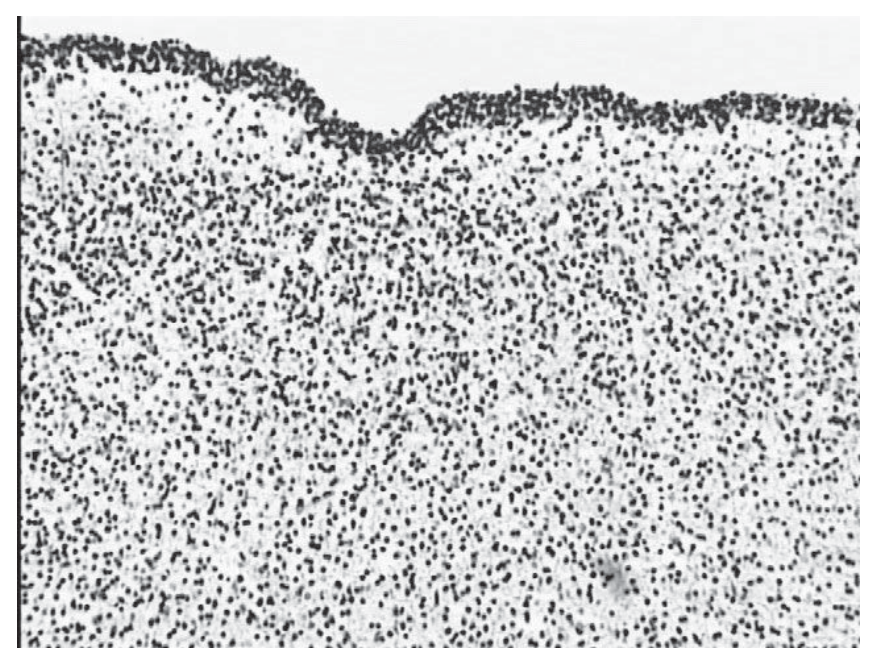

Fig. (4). Transversal section of cerebellar cortex with immature structure, uniformly made up of small round cells without the usual four-layered shape in a case of sudden neonatal death (magnification: 20x).

Table 2 shows the morphological alterations of the central autonomic nervous system observed in all the unexplained sudden death victims.

\section{Main Functional Findings in Brainstem and Cerebellum}

Using specific immmunohistochemical methods, altered expression of somatostatin, a neurotransmitter that is widely present in brainstem nuclei during fetal life but scarcely expressed after birth, was highlighted in the hypoglossus nucleus. Despite a normal structure, this nucleus was in fact somatostatin-immunonegative in $27 \%$ of the unexplained fetal deaths (17 cases) and, on the contrary, immunopositive in $44 \%$ of the SIDS victims ( 57 cases).

Negative expression of tyrosine-hydroxylase (TH), an essential enzyme in catecholamine biosynthesis, was detectable in a well-structured locus coeruleus (the major brainstem producer of noradrenaline) in $57 \%$ of the perinatal and infant sudden deaths (124 of the 217 cases).

We observed unusual apoptosis of the Purkinje cells and of the internal granular layer in the cerebellar cortex of 23 SIDS victims (18\%) and high apoptotic index in the dentate nucleus of 44 SIDS cases (34\%). A more in-depth description of these results can be found in our previous immunohistochemical works [15-17].

Frequently, two or more morphological and/or physiological alterations were observable in the same case, particularly in fetal deaths. In seven victims, we found the simultaneous presence of hypoplasia of different nuclei (the arcuate, pre-Bötzinger and parafacial nuclei) and of negative somatostatin expression in the hypoglossus nucleus.

None of the above reported alterations was found in the border-line population.

\section{DISCUSSION}

This study summarizes the results of our in-depth neuropathological researches performed in a 6-year period on a very wide, selected set of unexpected and unexplained perinatal deaths and SIDS, investigated following specific guidelines in accordance with the decree of the Lombardy Region [11]. The results hereafter indicated are, in part, reported in previous works [15-24].

The accurate examination of the central autonomic nervous system, allowed us to identify even minute alterations of the nuclei and/or structures overseeing the vital functions in these pathologies.

In particular, in $84 \%$ of all the unexplained deaths, we highlighted structural and/or functional alterations, particu-

Table 2. Morphological Alterations of the Brainstem and Cerebellum in Unexpected Death Victims

\begin{tabular}{|c|c|c|c|c|c|}
\hline $\begin{array}{l}\text { Unexpected death } \\
\text { victims }\end{array}$ & $\begin{array}{l}\text { Arcuate nucleus } \\
\text { hypoplasia }\end{array}$ & $\begin{array}{c}\text { Pre-Bötzinger nucleus } \\
\text { hypoplasia }\end{array}$ & $\begin{array}{l}\text { Parabrachial Kölliker- } \\
\text { Fuse complex hypoplasia }\end{array}$ & $\begin{array}{c}\text { Parafacial nucleus } \\
\text { hypoplasia }\end{array}$ & $\begin{array}{c}\text { Cerebellar cortex } \\
\text { immature structure }\end{array}$ \\
\hline $\begin{array}{c}\text { stillbirths } \\
\text { n/tot }(\%)\end{array}$ & $\begin{array}{c}33 / 59 \\
(56) \\
\end{array}$ & $\begin{array}{l}15 / 59 \\
(25)^{* \#}\end{array}$ & $\begin{array}{l}14 / 59 \\
(24)^{*}\end{array}$ & $\begin{array}{l}41 / 59 \\
(70)^{* \#}\end{array}$ & $\begin{array}{l}6 / 59 \\
(10) \\
\end{array}$ \\
\hline $\begin{array}{c}\text { newborns } \\
\text { n/tot(\%) }\end{array}$ & $\begin{array}{c}14 / 28 \\
(50)\end{array}$ & $0^{\#}$ & $\begin{array}{l}8 / 28 \\
(29) \#\end{array}$ & $0 *$ & $\begin{array}{l}6 / 28 \\
(20)\end{array}$ \\
\hline SIDS n/tot(\%) & $\begin{array}{c}65 / 130 \\
(50)\end{array}$ & $\begin{array}{c}10 / 130 \\
(8) *\end{array}$ & $0 * \#$ & $0^{\#}$ & $\begin{array}{c}34 / 130 \\
(26)\end{array}$ \\
\hline statistics & Not significant & $\begin{array}{c}\text { \# SB }>\text { NB p }=0.0030 \\
* \mathrm{SB}>\mathrm{SIDS} \mathrm{p}=0.0008\end{array}$ & $\begin{array}{c}* \mathrm{SB}>\mathrm{SIDS} \mathrm{p}<0.0001 \\
* \mathrm{NB}>\mathrm{SIDS} \mathrm{p}<0.0001\end{array}$ & $\begin{array}{c}* \mathrm{SB}>\mathrm{NB} \mathrm{p}>0.0001 \\
\quad \# \mathrm{SB}>\mathrm{SIDS} \mathrm{p}=0\end{array}$ & Not significant \\
\hline
\end{tabular}

SIDS=sudden infant death syndrome

One way ANOVA and Student's paired t-test were performed to compare the incidence of hypoplasia in the different districts of central autonomic nervous system. 
larly of the brainstem and cerebellum. These alterations represent a common morphological substrate in both unexplained perinatal death and SIDS and, therefore, can be considered congenital in nature.

A common finding was hypoplasia of the arcuate nucleus, a chemoreceptoral component of the ventral surface of the medulla oblongata, which was present in over $50 \%$ of all the victims, albeit with different degrees of extension and severity. In perinatal deaths, in addition to hypoplasia of the arcuate nucleus, we observed hypodevelopment of the dendritic fibers with a decreased neuronal density in the preBötzinger area, besides hypoplasia of the parabrachial/Kölliker-Fuse complex. The pre-Bötzinger nucleus has been physiologically defined as a group of neurons of the ventrolateral medulla, that are essential for generating the respiratory rhythm, as well as for modulating eupneic breathing $[24,25]$. Instead, the Kölliker-Fuse nucleus has an important function during intrauterine life, inhibiting the response of central and peripheral chemoreceptors (which are already fully formed and potentially functional) and therefore becomes any respiratory reflex. After birth, the Kölliker-Fuse abruptly reduces its inhibitory effects and becomes active as a respiratory center, able to coordinate the pulmonary motor responses to hematic oscillations of $\mathrm{pO}_{2}, \mathrm{pCO}_{2}$ and $\mathrm{pH}$ $[18,21]$.

In addition, exclusively in unexplained stillbirths, we observed very frequently (in 70\% of cases) the hypoplasia of the parafacial nucleus, consisting of "pre-inspiratory" neurons that periodically trigger the inspiratory neurons of the pre-Bötzinger complex [26,27]. Therefore, the function of hierarchical modulation of the breathing circuitry can be ascribed to the parafacial nucleus.

Among the functional alterations, we diagnosed altered expression of neurotransmitters in the brainstem, particularly of catecholamine in the locus coeruleus and somatostatin in the hypoglossus nucleus, besides defective apoptotic programs in the cerebellum, in the Purkinje and internal granular layers of the cerebellar cortex, as well as in the dentate nucleus [15-17].

The above reported findings indicate that both unexplained perinatal death and SIDS share common congenital anomalies of the central autonomic nervous system. Nevertheless, these alterations are wider and more severe in fetal deaths. In fact, whereas in SIDS victims the more frequent observation is hypoplasia of the arcuate nucleus, unexpected ante-partum deaths are characterized by hypodevelopment of various structures: hypoplasia of the arcuate nucleus, of the parabrachial/Kölliker-Fuse complex, of the pre-Bötzinger complex and of the parafacial nucleus. In particular, this last lesion represents a marker of unexpected ante-partum death. In fact it was found exclusively, and with a high frequency $(70 \%)$, in fetal deaths.

The distinctive congenital anomaly that marks the intrapartum death was shown to be the hypoplasia of the parabrachial/Kölliker-Fuse complex.

All these alterations, responsible for serious vagal reflexes, can be interpreted as expressions of a disturbed development of the nervous system in pathophysiological response to a variety of mechanisms and, above all, to mater- nal cigarette smoking in pregnancy, as we have previously suggested $[16,28,29]$.

In conclusion, the neuropathologic substrates common to both fetal and neonatal deaths can be ascribed to a process of unnatural selection caused by exogenous pathogenic noxae. The effects of these are particularly important, in view of their frequency and the variety of alterations observed, during fetal life. Therefore, unexpected perinatal loss should not be regarded as a separate entity from SIDS, since both are an integral part of autonomic nervous system abnormalities.

\section{ACKNOWLEDGEMENTS}

This study was supported by the Italian Lombardy Region target project n. 49210/2000 (Program of research and intervention for the reduction of the risk of SIDS and unexpected fetal death), by the agreement Lombardy RegionPfizer Italia S.r.l. n. 814/2006, and by Ministry of Foreign Affairs (joined projects of particular relevance "Anatomopathologic and genetic study of the unexplained perinatal death and SIDS") n. 269/P/0085087/2004 and n. 0083227/20006.

\section{REFERENCES}

[1] World Health Organization (WHO). Neonatal and perinatal mortality: country, regional and global estimates. 2006; 1-75.

[2] Cartlidge PHT, Dawson AT, Stewart JH, Vujanic GM. Value and quality of perinatal and infant postmortem examination: cohort analysis of 400 consecutive deaths. BMJ 1995; 310: 155-8.

[3] Stanton C, Lawn JE, Rahman H, Wilczynska-Ketende K, Hill K. Stillbirth rates: delivering estimates in 190 countries. Lancet 2006; 367: 1487-94.

[4] McClure EM, Nalubamba-Phiri M, Goldenberg RL. Stillbirth in developing countries. Int J Gynaecol Obstet 2006; 94: 82-90.

[5] Magee JF. Investigation of stillbirth. Pediatar Dev Pathol 2001; 4: $1-22$.

[6] Malloy MH, MacDorman M. Changes in the classification of sudden unexpected infant deaths: United States, 1992-2001. Pediatrics 2005; 115: 1247-53.

[7] Grafe MR, Kinney HC. Neuropathology associated with stillbirth. Semin Perinatol 2002; 26: 83-8.

[8] Bell JE, Becker JC, Wyatt B, Keeling JW, McIntosh N. Brain damage and axonal injury in a Scottish cohort of neonatal deaths. Brain 2005; 128: 1070-81.

[9] Squier M, Keeking JW. The incidence of prenatal brain injury. Neuropathol Appl Neurobiol 1991; 17: 29-38.

[10] Sparks DL, Hunsaker JC. Neuropathology of sudden infant death (syndrome). Childs Nerv Syst 2002; 18: 568-92.

[11] Lombardy Regional Decree n. 11693 of 06-20-2002 "Legislative measures on adoption of anatomo-pathological and forensicpathological intervention targeted on the prevention, knowledge, and identification of the sudden infant death cases".

[12] Matturri L, Ottaviani G, Alfonsi G, Crippa M, Rossi L, Lavezzi AM. Study of the brainstem, particularly the arcuate nucleus, in sudden infant death syndrome (SIDS) and sudden intrauterine unexplained death (SIUD). Am J Forensic Med Pathol 2004; 25: 44-8.

[13] Matturri L, Ottaviani G, Lavezzi AM. Techniques and criteria in pathologic and forensic-medical diagnostics of sudden unexpected infant and perinatal death. Am J Clin Pathol 2005; 124: 259-68.

[14] Matturri L, Ottaviani G, Lavezzi AM, Guidelines for neuropathologic diagnostics of perinatal unexpected loss and sudden infant death sindrome (SIDS). A technical protocol. Virchows Arch 2008; 452: 19-25.

[15] Lavezzi AM, Ottaviani G, Matturri L. Role of somatostatin and apoptosis in breathing control in sudden perinatal and infant unexplained death. Clin Neuropathol 2004; 23: 304-10.

[16] Lavezzi AM, Ottaviani G, Mingrone R, Matturri L. Analysis of the human locus coeruleus in perinatal and infant sudden unexplained death. Possible role of the cigarette smoking in the development of this nucleus. Dev Brain Res 2005; 154: 71-80.

[17] Lavezzi AM, Ottaviani G, Mauri M, Matturri L. Alterations of biological features of the cerebellum in sudden perinatal and infant death. Curr Mol Med 2006; 6: 429-35. 
[18] Lavezzi AM, Ottaviani G, Rossi L, Matturri L. Cytoarchitectural organization of the parabrachial/Kölliker-Fuse complex in man. Brain Dev 2004; 26: 316-20.

[19] Matturri L, Minoli I, Lavezzi AM, et al. Hypoplasia of meduallary arcuate nucleus in unexpected late fetal death (stillborn infants): a pathologic study. Pediatrics 2002; 109: E43.

[20] Matturri L, Biondo B, Suarez-Mier MP, Rossi L. Brain stem lesions in the sudden infant death syndrome: variability in the hypoplasia of the arcuate nucleus. Acta Neuropathol (Berl) 2002; 104: $12-20$.

[21] Lavezzi AM, Ottaviani G, Ballabio GM, Rossi L, Matturri L. Preliminary study on the cytoarchitecture of the human parabrachial/Kölliker-Fuse complex with reference to sudden infant death syndrome and sudden intrauterine unexplained death. Pediatr Dev Pathol 2004; 7: 171-9.

[22] Lavezzi AM, Ottaviani G, Rossi L, Matturri L. Hypoplasia of the Parabrachial/Kölliker-Fuse complex in perinatal death. Biol Neonate 2004; 86: 92-7.

[23] Lavezzi AM, Ottaviani G, Terni L, Matturri L. Histological and biological developmental characterization of the human cerebellar cortex. Int J Dev Neurosci 2006; 24: 365-71.
[24] Lavezzi AM, Matturri L. Functional neuroanatomy of the human pre-Bötzinger complex with particular reference to sudden unexplained perinatal and infant death. Neuropathology 2008; 28:10-6.

[25] Rekling JC, Feldman JL. PreBötzinger complex and pacemaker neurons: hypothesized site and kernel for respiratory rhythm generation. Annu Rev Physiol 1998; 60: 385-405.

[26] Chatonnet F, Borday C, Wrobel L, et al. Ontogeny of central rhythm generation in chicks and rodents. Respir Physiol 2006; 154: $37-56$.

[27] Guyenet PG. Novel two-rhythm generator theory of breathing in mammals. J Physiol 2006; 570: 407-20.

[28] Lavezzi AM, Ottaviani G, Mauri M, Matturri L. Hypoplasia of the arcuate nucleus and maternal smoking during pregnancy, in perinatal and infant sudden unexpected death. Neuropathology 2003; 24: 284-9.

[29] Lavezzi AM, Ottaviani G, Matturri L. Adverse effects of prenatal tabacco smoke exposure on biological parameters of the developing brainstem. Neurobiol Dis 2005; 20: 601-7.

(C) Matturri et al.; Licensee Bentham Open.

This is an open access article licensed under the terms of the Creative Commons Attribution Non-Commercial License (http://creativecommons.org/licenses/by-nc/3.0/) which permits unrestricted, non-commercial use, distribution and reproduction in any medium, provided the work is properly cited. 